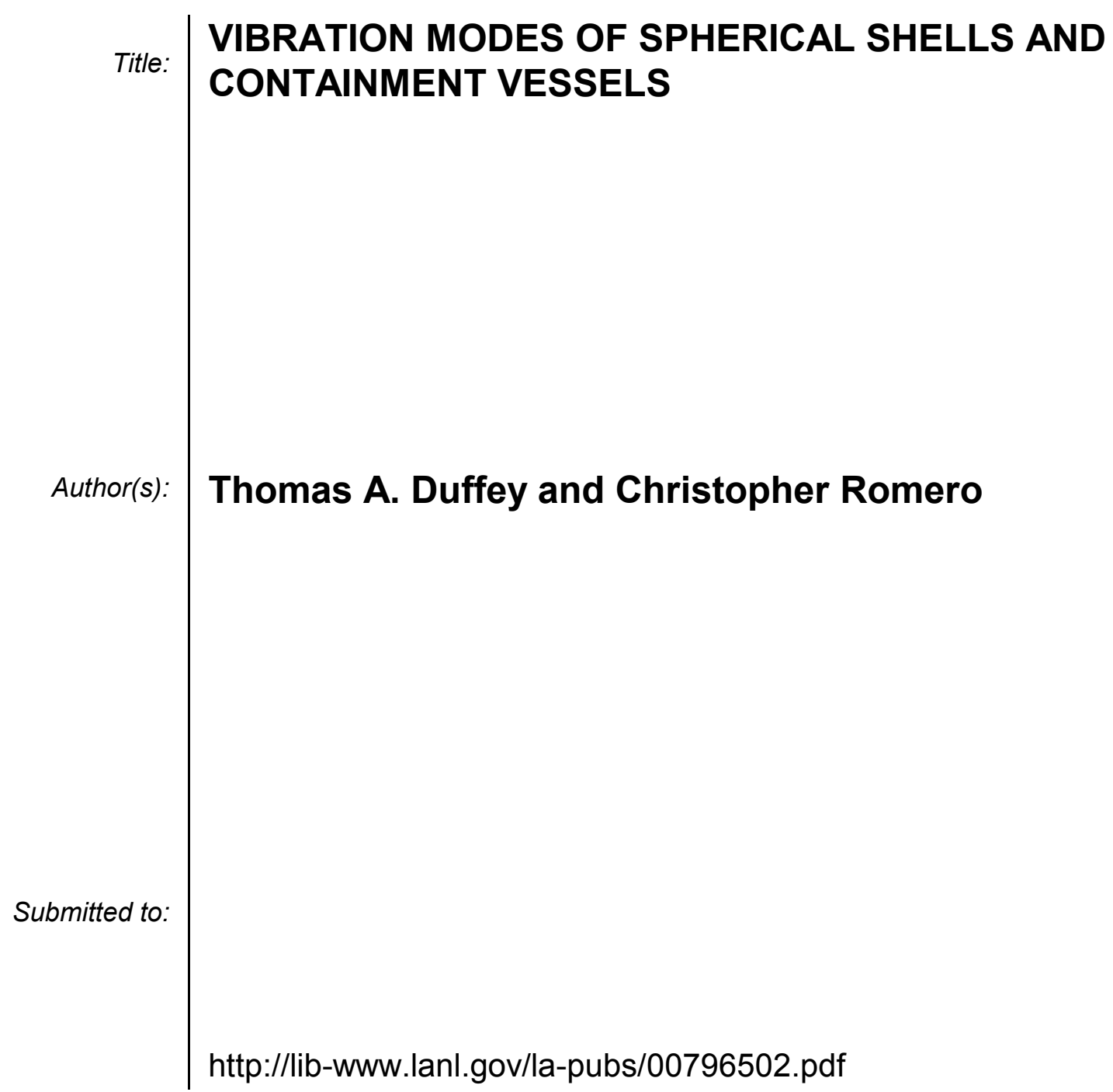




\section{VIBRATION MODES OF SPHERICAL SHELLS AND CONTAINMENT VESSELS}

\author{
Thomas A. Duffey \\ Consulting Engineer \\ PO Box 1239 \\ Tijeras, New Mexico 87059 \\ TDuffey2@aol.com \\ (505) 281-1241
}

\author{
Christopher Romero \\ Group DX-5 MS D411 \\ Los Alamos National Laboratory \\ Los Alamos, New Mexico 87545 \\ cromero@lanl.gov \\ (505) 665-6250
}

\begin{abstract}
Spherical pressure vessels are used to fully contain the effects of high explosions. In this paper, the vibrations of a spherical containment vessel undergoing elastic response are investigated. Vibration modes of containment vessels are of particular interest, as it is the superposition and interaction of different modes of response with closely spaced frequencies that has been reported to be the mechanism of 'strain growth'. First, the modal frequencies of a spherical shell for both axisymmetric and nonaxisymmetric response modes are discussed, based on a sequence of papers that have appeared in the open literature. Analytical predictions are then compared with numerical simulations using ABAQUS. It is found that the numerical simulations accurately predict both the axisymmetric and nonaxisymmetric modal frequencies for the complete spherical shell. Next, numerical simulations of modal frequencies for the more complex spherical containment vessel (with nozzles) are compared with the spherical shell results.

Numerical simulations for the spherical containment vessel reveal that frequencies are somewhat similar to the complete spherical shell. Limited comparisons with experimentally recorded frequencies for participating modes of vessel dynamic response during high explosive containment testing are presented as well.
\end{abstract}

\section{INTRODUCTION}

Spherical pressure vessels used to fully contain the effects of high explosions generally fall into one of two categories: 1 . Vessels designed for multiple use, in which the dynamic response of the vessel is restricted to the elastic range (e.g., see [1]); and 2. Vessels designed for one-time use only, in which the vessel may undergo substantial permanent plastic deformations (e.g., see [2]). In this paper, the vibrations of a spherical containment vessel are investigated for vessel response in the elastic range. These vibration modes, and particularly the spacing of natural frequencies, have recently been shown to be the mechanism of the 'strain growth' phenomenon [3]. 'Strain growth' is a phenomenon in which the maximum vessel response amplitude occurs later in time, i.e., well beyond the first response peak and in which the strain amplitude oscillates with time over an extended period (amplitude modulation caused by modal beating effects).

A typical spherical containment vessel, with nozzles, is shown in Fig. 1. Vibrations of such a containment vessel are related to those of a complete spherical shell. Therefore, a review of the literature on vibrations of a complete spherical shell is first presented in the next section, followed by a comparison of natural frequencies of axisymmetric (Section 3) and nonaxisymmetric (Section 4) modes of a complete spherical shell with ABAQUS finite element predictions. Corresponding natural frequencies of the more complex, spherical containment vessel are also compared in Section 4. Limited frequency comparisons with experimental results are presented in Section 5 , followed by a Conclusions section.

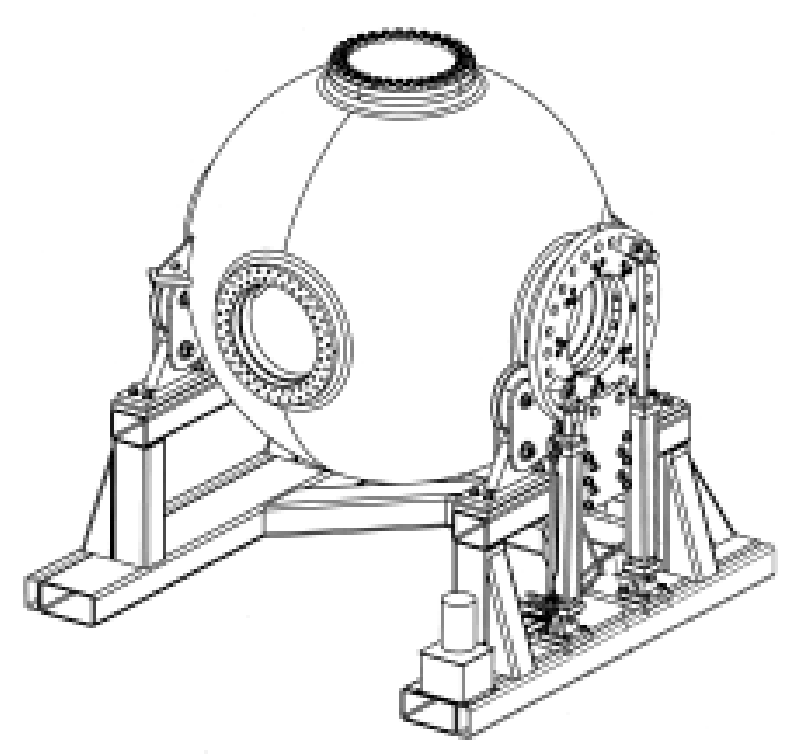

Figure 1. Typical Spherical High Explosive Containment Vessel 


\section{LITERATURE SURVEY}

Baker [4] extended the early work of H. Lamb [5] to determine the axisymmetric vibration modes of a thin complete spherical shell. He utilized a membrane shell theory and observed two types of vibration mode sets, falling on a lower and an upper branch. The lower branch was found to consist of an infinite number of modes, spaced within a finite frequency interval. Baker also demonstrated by experiment that the two types of vibration modes do in fact exist.

Silbiger [6] presented a brief discussion of the presence of nonaxisymmetric modes of spherical shells, using Baker's [4] two-branch, membrane solution as a basis. Silbiger states that nonaxisymmetric modes do indeed exist and that the corresponding frequencies are identical to the frequencies of the axisymmetric modes. He goes on to state that, for each branch, one can select only $2 \mathrm{n}+1$ linearly independent nonaxisymmetric modes, all other modes being linear combinations of the $2 n+1$ modes.

Kalnins [7] again examined axisymmetric modes of a complete spherical shell, but included the effects of bending. $\mathrm{He}$ also found that the frequency spectrum consists of two infinite sets of modes. He labeled one branch as flexural and the other as membrane, the distinction made on the basis of the comparison of strain energies due to bending and stretching of each mode ${ }^{1}$. However, he observed a fundamental difference in the lower branch behavior, as compared to Baker [4], due to the inclusion of bending. He showed that the lower branch modes are a degenerate case of bending modes in the membrane theory, applicable only to zero thickness shells. When Kalnins introduced the bending theory, the frequency interval for this lower branch was found to extend to infinity for every non-zero thickness shell (rather than all modes being spaced within a finite interval of the frequency spectrum). The upper (membrane) branch was nominally identical to that of Baker.

Niordson [8] rederived the equations for bending vibrations of a spherical shell in a somewhat different form. He examined the nonaxisymmetric modes as well, concluding that there exist $\mathrm{n}+1$ (not $2 \mathrm{n}+1$ as reported by Silbiger) modes at each frequency on each branch. Observations in [8] made regarding nonaxisymmetric modes (e.g., the fact that they exhibit the frequency degeneracy identified by Silbiger [6]) were similar, except for the number of independent modes anticipated.

Wilkinson [9] again investigated axisymmetric modes of a complete spherical shell, but with the effects of transverse shear and rotatory inertia included. Not surprisingly, he observed a third branch at higher frequencies because of the inclusion of shear deformations.

Shah, et al. [10] investigated nonaxisymmetric wave propagation in a hollow elastic sphere. They presented a shell theory in which the effects of transverse normal strain are

\footnotetext{
${ }^{1}$ A closed shell cannot undergo pure bending. A mode is either purely extensional (membrane) or combined bending-extensional, but can (on an energy basis) be "mostly" bending. Stated another way, no modes will be present for which the extension of the middle surface is identically zero.
}

included as are transverse shear and rotary inertia. This represents a progressive extension of earlier work cited above. Shah, et al., observed the presence of axisymmetric modes which are independent of the circumferential angle. They then state that, by a suitable superposition of the axisymmetric modes of vibration about different axes, but of identical natural frequency, a nonaxisymmetric vibration mode of the same frequency results, in agreement with observations made by Silbiger [6] and Niordson [8].

Hirai and Kuroda [11] investigated the natural vibrations of a spherical shell of variable thickness in the meridional direction. They investigated nonaxisymmetrical vibrations. Unfortunately, results were only presented for a hemispherical shell. Furthermore, no general information regarding the role played by nonaxisymmetrical modes was given.

Evans [12] investigates nonaxisymmetric modes of a complete spherical shell, placing his results in terms of modal impedances. The work is limited to extensional effects for a thin shell, i.e., membrane theory only. Evans claims that Silbiger [6] overlooked the fact that there is a lack of orthogonality of certain of the nonaxisymmetric modes. Evans does, however, confirm Silbiger's observation that natural frequencies of nonaxisymmetric modes agree with their axisymmetric mode counterparts, i.e., that natural frequencies of the nonaxisymmetric modes are degenerate.

Useful numerical results on the vibration of complete spherical shells are presented in the ABAQUS/Standard Example Problems Manual [13]. Both axisymmetric and nonaxisymmetric comparisons are presented. For nonaxisymmetric modes, the first 20 eigenvalues were investigated. Indeed, as predicted by Silbiger [6], $2 n+1$ linearly independent modes were recovered corresponding to each value of $\mathrm{n}$ investigated.

\section{AXISYMMETRIC MODES OF VIBRATION}

Axisymmetric modes are independent of the circumferential angle, $\theta$ (See Fig. 2). A detailed discussion of axisymmetric membrane modes of a complete spherical shell is presented in [4]. Modes of vibration are expressed in terms of Legendre Polynomials of integer indices, $n$. For each value of $n \geq 2$, there are two branches, i.e., two separate, but similar, mode shapes, and two distinct frequencies (For $\mathrm{n}=0$, there is only one real root, corresponding to the 'fundamental' membrane or 'breathing' mode; $\mathrm{n}=1$ corresponds to purely rigid-body displacement, i.e., no distortions). The lower branch approaches an asymptotic value for large $\mathrm{n}$, as described in [4], which unfortunately appears to be physically meaningless because the intervals between the natural frequencies apparently become increasingly small [7].

The elementary bending/membrane theory developed in [7] has a similar upper branch when compared against the membrane theory. However, the lower branch monotonically increases with integer index number when bending is included. 
Natural frequencies for these axisymmetric bending/membrane modes are plotted as a function of index number in Fig. 3 using the following geometry and material parameters relevant to the example spherical shell/containment vessel introduced later in the paper:

$$
\begin{array}{ll}
\text { Shell thickness } & \mathrm{h}=2.00 \text { in } \\
\text { Mean radius } & \mathrm{R}=37.0 \text { in } \\
\text { Elastic modulus } & \mathrm{E}=29.9 \times 10^{6} \mathrm{psi} \\
\text { Mass density } & \mu=7.32 \times 10^{-4} \mathrm{lbf}-\mathrm{sec}^{2} / \mathrm{in}^{4} \\
\text { Poisson's ratio } & \nu=0.29
\end{array}
$$
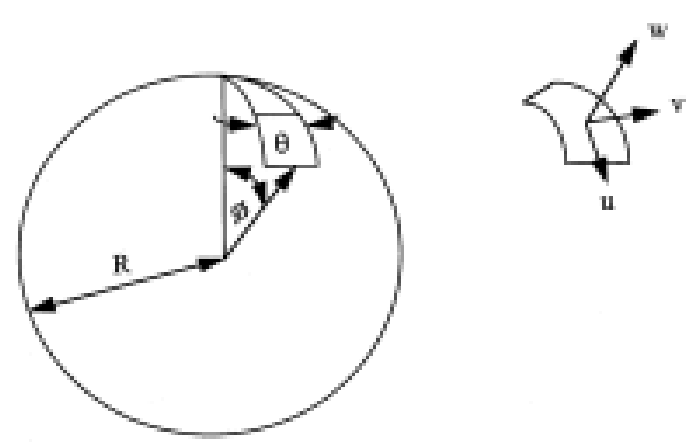

Figure 2. Complete Spherical Shell

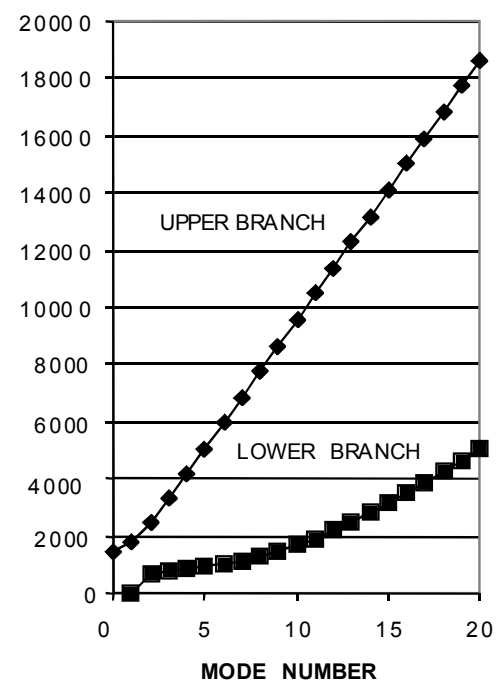

Figure 3. Natural Frequencies for a Complete Spherical Shell
The upper and lower branches can be clearly seen in Fig. 3 .

In the higher-order theory [9], which includes transverse shear and rotary inertia, a third, highest branch appears, associated with the shear-deformation degree of freedom introduced. However, this highest branch is of little interest here because the frequencies are so high. The lower and upper branches nominally agree with the elementary bending/membrane theory. Therefore, attention is given to the lower and upper branches in this paper.

A finite element model of a complete spherical shell using axisymmetric elements was developed for use with ABAQUS, using the above shell parameters. The first 26 modes extracted by ABAQUS are compared with the lowest 26 modes predicted by the higher-order theory of Wilkinson [9] in Table 1, from which the following observations can be made:

1. The first 26 modes (in ascending numerical order) draw from both lower and upper branches.

2. The lowest mode (zero frequency) is the rigid-body mode, associated with the lower branch.

3. The so-called 'fundamental' mode is actually the tenth mode to appear. It is, however, the first mode of the upper branch.

4. The percent difference in respective theoretical and ABAQUS-predicted frequencies is extremely small, in all cases much less than one percent.

5. Some of the frequencies are very close in value. For example, modes 9 and 10 have frequencies within $1 \mathrm{~Hz}$, owing to the fact that the modes are associated with different branches. Modes 15 and 16 and modes 25 and 26 are also quite close. The implication is that beating effects could occur, resulting in the "strain growth" phenomenon [3]. Such effects were observed in experiments on complete spherical shells reported by Baker [4] and are the subject of a recent investigation by Whenhui, et al. [3]. 
TABLE 1

NATURAL FREQUENCY COMPARISON - AXISYMMETRIC MODES

\begin{tabular}{|c|c|c|c|c|c|}
\hline MODE & ABAQUS & THEOR. & INDEX, $\mathbf{n}$ & BRANCH & PCT. DIFF. \\
\hline 1 & $1.32 \mathrm{E}-4$ & 0.0 & 1 (Rigid Body) & Lower & --- \\
\hline 2 & 642.77 & 642.84 & 2 & Lower & 0.011 \\
\hline 3 & 768.69 & 769.36 & 3 & Lower & 0.087 \\
\hline 4 & 837.87 & 839.39 & 4 & Lower & 0.181 \\
\hline 5 & 906.61 & 909.33 & 5 & Lower & 0.299 \\
\hline 6 & 995.67 & 999.55 & 6 & Lower & 0.388 \\
\hline 7 & 1114.5 & 1119.6 & 7 & Lower & 0.456 \\
\hline 8 & 1266.6 & 1273.3 & 8 & Lower & 0.526 \\
\hline 9 & 1451.8 & 1459.8 & 9 & Lower & 0.548 \\
\hline 10 & 1461.3 & 1460.7 & 0 (Fundamental) & Upper & 0.041 \\
\hline 11 & 1668.2 & 1676.2 & 10 & Lower & 0.477 \\
\hline 12 & 1787.9 & 1787.2 & 1 & Upper & 0.039 \\
\hline 13 & 1913.3 & 1921.8 & 11 & Lower & 0.442 \\
\hline 14 & 2184.3 & 2192.8 & 12 & Lower & 0.388 \\
\hline 15 & 2466.4 & 2467.5 & 2 & Upper & 0.045 \\
\hline 16 & 2478.9 & 2487.5 & 13 & Lower & 0.346 \\
\hline 17 & 2794.8 & 2802.2 & 14 & Lower & 0.264 \\
\hline 18 & 3130.2 & 3136.9 & 15 & Lower & 0.214 \\
\hline 19 & 3296.0 & 3297.9 & 3 & Upper & 0.058 \\
\hline 20 & 3483.4 & 3488.0 & 16 & Lower & 0.132 \\
\hline 21 & 3852.7 & 3855.4 & 17 & Lower & 0.070 \\
\hline 22 & 4169.1 & 4172.8 & 4 & Upper & 0.089 \\
\hline 23 & 4236.9 & 4237.4 & 18 & Lower & 0.012 \\
\hline 24 & 4634.7 & 4632.1 & 19 & Lower & 0.056 \\
\hline 25 & 5045.1 & 5038.7 & 20 & Lower & 0.127 \\
\hline 26 & 5057.1 & 5062.3 & 5 & Upper & 0.103 \\
\hline
\end{tabular}

\section{NONAXISYMMETRIC MODES OF VIBRATION}

As discussed primarily in [6], nonaxisymmetric modes for a complete spherical shell do exist. Nonaxisymmetric modes depend upon both $\theta$ and $\phi$ (See Fig. 2). They are degenerate: frequencies are identical to corresponding frequencies of axisymmetric modes. Silbiger [6] attributes this to the spherical symmetry of the shell. He argues that the axisymmetric modes are defined with respect to a specific set of axes. Due to the symmetry of the shell, however, it can vibrate in similar modes with a different axis orientation. For a given set of "identical' modes (differing only in orientation), the modes will each have the same natural frequency. These axisymmetric modes of identical natural frequency can be superimposed to obtain new nonaxisymmetric modes that have the same natural frequency as the corresponding axisymmetric mode, but which are not symmetric with respect to any axis. Silbiger [6] goes on to state that, corresponding to each natural frequency, there exist $2 n+1$ linearly independent modes, all other modes (at that frequency) being linear combinations of these modes. As discussed earlier, Niordson [8], apparently unaware of the work of Silbiger, predicted that for each integer $\mathrm{n}>1$, there is one axisymmetric mode and $\mathrm{n}$ nonaxisymmetric modes, leading to $\mathrm{n}+1$ (rather than $2 \mathrm{n}+1$ ) modes at the same natural frequency. His physical explanation of the degeneracy was, however, similar to that of Silbiger.

Actual spherical containment vessels contain deviations from spherical symmetry due to the presence of nozzles, not to mention significant manufacturing variations. Silbiger [6] points out that when such a deviation from spherical symmetry 
occurs, the above degeneracy is no longer present, and the particular modal frequency for generic integer index, $n$, on one of the solution branches will split into $2 n+1$ distinct frequencies. In a later paper, Niordson [14] similarly indicates that when spherical symmetry of a complete spherical shell is lost, the previously degenerate frequencies form into bands, with the bandwidth related to the degree of spherical asymmetry.

Two additional models were constructed and run on ABAQUS/Standard. The first was a full model of a complete spherical shell; the second was a full model of the spherical containment vessel, including ports. The expectation was that additional nonaxisymmetric modes would appear in both these cases, as the axisymmetric restriction of the spherical shell described in the previous section had been removed. A total of 200 normal modes of vibration were extracted using ABAQUS. Results for a limited number of selected modes are shown in Table 2. Contents of the eight columns of Table 2 are as follow:

Col. 1: This is the number of the mode extracted, in order of increasing frequency (This is not the integer index, $n$ ).

Col. 2: This is the frequency (in $\mathrm{Hz}$ ) predicted from the appropriate axisymmetric solution [9]. In addition, because of the relaxation of the axisymmetric boundary conditions, torsional modes can occur. These torsional modes were determined from [15]. Note further in Col. 2 that there are sets of "duplicate" frequencies, as predicted by Silbiger [6]. These correspond to the $2 n+1$ linearly independent nonaxisymmetric modes expected for each value of the integer index, $n$, for each branch.

Col.3: This is the appropriate value of the integer index, $\mathrm{n}$, for the frequency value listed. This index was identified by comparing the ABAQUS-extracted frequency value of interest with predictions of the various branches of the bendingextensional (radial-tangential) modes given by Wilkinson [9] or the torsional modes [15]. Note that, in some cases for the particular geometry and material properties considered, frequencies of different modes overlap and there is some question of precise identification of the type, branch, and integer index value, $n$. While the precise identification of each mode does not appear essential here, what is significant is that modes in some cases are extremely closely spaced, possibly leading to a beating phenomenon and amplification of subharmonics.

Col 4: This column identifies the applicable branch of the observed modes.

Col. 5: This column gives the expected number of linearly independent modes, $2 \mathrm{n}+1$, as predicted by Silbiger [6].

Col. 6: These are the ABAQUS-extracted normal-mode frequencies.

Col. 7: This column lists the number of observed modes from ABAQUS. A comparison of columns 5 and 7 indicates that the number of modes extracted by ABAQUS agrees precisely with the number of theoretical nonaxisymmetric modes. Examination of corresponding theoretical axisymmetric frequency values (Col. 2) with ABAQUS-calculated values indicates that agreement is excellent, at least for the lower values of $n$ shown.

Col. 8: This column contains corresponding nonaxisymmetric ABAQUS modal extraction results for the full vessel model (including nozzles). Frequencies are seen to be somewhat similar to the nonaxisymmetric shell results, although because of the true asymmetry in the model caused primarily by the presence of the nozzles, each mode has a distinct frequency. This is precisely the behavior predicted by Silbiger [6]: $\mathrm{He}$ predicted that the degeneracy would be removed, and $2 n+1$ distinct frequencies for each integer index, $n$, would appear for each branch. 
TABLE 2

SELECTED NONAXISYMMETRIC COMPARISONS

\begin{tabular}{|c|c|c|c|c|c|c|c|}
\hline MODE & $\begin{array}{c}\text { REF. [9], } \\
{[15]}\end{array}$ & $\underset{\mathbf{n}}{\text { INDEX, }}$ & BRANCH & $\begin{array}{l}\text { EXPECTED } \\
\text { NUMBER }\end{array}$ & $\begin{array}{l}\text { ABAQUS } \\
\text { FULL }\end{array}$ & $\begin{array}{l}\text { OBSERVED } \\
\text { NUMBER }\end{array}$ & $\begin{array}{c}\text { CONTAINMENT } \\
\text { VESSEL }\end{array}$ \\
\hline $1-3$ & 0 & $1(\mathrm{RB})$ & Lower & 3 Modes & 0 & 3 Modes & 0 \\
\hline $4-6$ & 0 & $1(\mathrm{RB})$ & Torsion & 3 Modes & 0 & 3 Modes & 0 \\
\hline 7 & 642.84 & 2 & Lower & & 643.39 & & 541.95 \\
\hline 8 & 642.84 & 2 & Lower & & 643.40 & & 586.41 \\
\hline 9 & 642.84 & 2 & Lower & & 643.41 & & 605.16 \\
\hline 10 & 642.84 & 2 & Lower & & 643.47 & & 606.99 \\
\hline \multirow[t]{2}{*}{11} & 642.84 & 2 & Lower & & 643.48 & & 635.29 \\
\hline & & & & 5 Modes & & 5 Modes & \\
\hline & & & & & & & \\
\hline 12 & 769.36 & 3 & Lower & & 770.23 & & 663.81 \\
\hline 13 & 769.36 & 3 & Lower & & 770.24 & & 701.76 \\
\hline 14 & 769.36 & 3 & Lower & & 770.27 & & 712.79 \\
\hline 15 & 769.36 & 3 & Lower & & 770.49 & & 723.1 \\
\hline 16 & 769.36 & 3 & Lower & & 770.64 & & 736.04 \\
\hline 17 & 769.36 & 3 & Lower & & 770.92 & & 750.25 \\
\hline \multirow[t]{2}{*}{18} & 769.36 & 3 & Lower & & 771.10 & & 758.81 \\
\hline & & & & 7 Modes & & 7 Modes & \\
\hline 89 & 1460.7 & 0 & Upper & 1 Mode & 1462.8 & 1 Mode & 1421.5 \\
\hline 137 & 1787.2 & 1 & Upper & & 1791.6 & & 1818.0 \\
\hline 138 & 1787.2 & 1 & Upper & & 1792.0 & & 1865.6 \\
\hline \multirow[t]{2}{*}{139} & 1787.2 & 1 & Upper & & 1792.2 & & 1891.3 \\
\hline & & & & 3 Modes & & 3 Modes & \\
\hline
\end{tabular}

A comparison of spherical natural frequencies with the spherical containment vessel (with nozzles) for the first 200 modes is shown in Fig. 4.

\section{EXPERIMENTAL OBSERVATIONS}

The existence of 'lower branch' modes for dynamically loaded spherical vessels was first reported by Baker [4]. Both lower- and upper-branch participating modes were also reported in [16] on a vessel similar to that investigated herein. The two dominant (participating) modes were found to be the fundamental membrane mode $(1397 \mathrm{~Hz})$ and a lower branch mode at approximately $1100 \mathrm{~Hz}$, based upon an FFT of a single strain-time record taken on the vessel outer surface. Moreover, Martineau [17] also performed an FFT of strain-time data recorded during the dynamic (High Explosive) response process

on the outer surface of the vessel analyzed in this paper. $\mathrm{He}$ found several participating modes, as shown in Table 3 . Comparisons with modal frequencies from ABAQUS calculations are also indicated in Table 3. Note that, with the exception of the 'fundamental' mode the precise identification of the participating mode in Table 3 is not necessarily assured in view of finite-element modeling approximations taken, including the neglect of the rather significant spatial variation in the vessel thickness due to the manufacturing process. It is clear, however, that modes from both lower and upper branches significantly participate in the transient vessel response, with several participating modes below the so-called 'fundamental' mode. 
TABLE 3

A COMPARISON OF OBSERVED PARTICIPATING MODES

\begin{tabular}{|c|c|c|}
\hline $\begin{array}{c}\text { EXPERIMENTAL } \\
\text { FREQUENCY, HZ }\end{array}$ & $\begin{array}{c}\text { ESTIMATED } \\
\text { BRANCH }\end{array}$ & $\begin{array}{c}\text { ESTIMATED } \\
\text { MODE } \\
\text { NUMBER, N }\end{array}$ \\
\hline $\mathbf{8 3 6}$ & Lower & $\mathbf{5}$ \\
\hline $\mathbf{9 3 6}$ & Lower & $\mathbf{6}$ \\
\hline 1025 & Lower & Unknown \\
\hline 1169 & Lower & 7 \\
\hline 1357 & Upper & $\begin{array}{c}\text { 0 } \\
\text { ('Fundamental') }\end{array}$ \\
\hline 1556 & Lower or Upper & Unknown \\
\hline
\end{tabular}

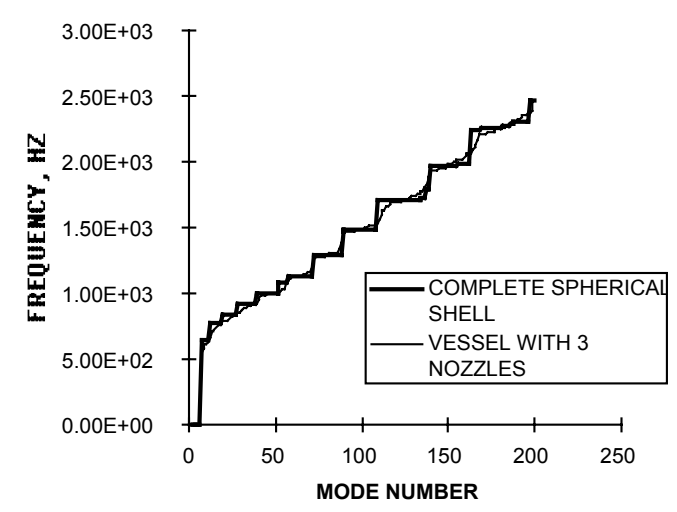

Figure 4. Frequency Comparison for Complete Spherical Shell Model and Spherical Containment Vessel

\section{CONCLUSIONS}

1. Based upon comparisons of theoretical predictions and axisymmetric finite element simulations, ABAQUS predicts the axisymmetric natural frequencies of a spherical shell with high accuracy (The first 26 modes of the example investigated were all well within one percent of corresponding theoretical values).

2. Corresponding nonaxisymmetric modes of a complete spherical shell were also accurately predicted by ABAQUS. Results confirmed the existence of the $2 n+1$ linearly independent, degenerate modes at each discrete frequency value. These degenerate modes, according to Silbiger, have identical mode shape, but are independent because an axis of symmetry cannot be uniquely be specified for a complete, free spherical shell.

3. Calculations using ABAQUS for a similar containment vessel with nozzles revealed that frequencies were somewhat similar, as expected. However, each modal frequency was found to be distinct, as predicted by Silbiger, suggesting that mode shapes of nonaxisymmetric modes are unique in this case.

\section{ACKNOWLEDGMENTS}

This work was performed for the Los Alamos National Laboratory under Contract No. W-7405-ENG-36 with the US Department of Energy (DOE).

\section{REFERENCES}

1. J.J. White and B.D. Trott, "Scaling Law for the Elastic Response of Spherical Explosion-Containment Vessels", Experimental Mechanics, Vol. 20, No. 5, pp. 174-177 (1980).

2. W.E. Baker, "The Elastic-Plastic Response of thin Spherical Shells to Internal Blast Loading", Journal of Applied Mechanics, Vol. 27, pp. 139-144 (1960).

3. A. Whenhui, X. Honglu, A. Guangquan and G.K. Schleyer, "Dynamic Response of Cylindrical Explosive Chambers to Internal Blast Loading Produced by a Concentrated Charge", International Journal of Impact Engineering, Vol. 19, pp. 831-845 (1997).

4. W.E. Baker, "Axisymmetric Modes of Vibration of Thin Spherical Shell", Journal of the Acoustical Society of America, Vol. 33, pp. 1749-1758 (1961).

5. H. Lamb, "On the Vibrations of a Spherical Shell", Proceedings of the London Mathematical Society, Vol. XIV, p. 50 (1883).

6. A. Silbiger, "Nonaxisymmetric Modes of Vibration of Thin Spherical Shells", Journal of the Acoustical Society of America, Vol. 38, pp. 367-368 (1965).

7. A. Kalnins, "Effect of Bending on Vibrations of Spherical Shells", Journal of the Acoustical Society of America, Vol. 36, pp. 74-81 (1964).

8. F.I. Niordson, "Free Vibrations of Thin Elastic Spherical Shells", International Journal of Solids and Structures. Vol. 20, pp. 667-687 (1984).

9. J.P. Wilkinson, "Natural Frequencies of Closed Spherical Shells", ", Journal of the Acoustical Society of America, Vol. 38, pp. 367-368 (1965).

10. A.H. Shah, C.V. Ramkrishnan, and S.K. Datta, "ThreeDimensional and Shell-Theory Analysis of Elastic Waves in a Hollow Sphere", Journal of Applied Mechanics, Vol. 36, pp. 431-439 (1969).

11. T. Hirai and M. Kuroda, "Analysis of Natural Vibrations of a Spherical Shell with Variable Thickness", Journal of the Acoustical Society of America, Vol. 86, pp. 1864-1875 (1989).

12. R.B. Evans, "Modal Impedances for Nonaxisymmetric Vibrations of a Thin Spherical Shell", Journal of the Acoustical Society of America, Vol. 100, pp. 1242-1245 (1996). 
13. Anon., ABAQUS/Standard Example Problems Manual, Hibbitt, Karlsson and Sorenson, Inc., pp. 3.1.1-1 - 8 (1997).

14. F.I. Niordson, "The Spectrum of Free Vibrations of a Thin Elastic Spherical Shell", International Journal of Solids and Structures, Vol. 24, pp. 947-961 (1988).

15. R.D. Blevins, Formulas for Natural Frequency and Mode Shape, Krieger Publishing Co., pp. 328-330 (1984).

16. T.A. Duffey, W.E. Baker, B.B. Lewis and J.M. Greene, "Containment of Explosions in Spherical Shells", Presented at ASME Pressure Vessel and Piping Conference, July 1993, Piping Supports and Structural Dynamics (A. Dermenjian, Ed.), ASME PVP Vol. 264 (1993).

17. R. Martineau, Los Alamos National Laboratory, Personal Communication, May 27, 1999. 